\title{
Value-based Healthcare
}

\section{Value-based Healthcare: Patient-reported Outcomes in Clinical Decision Making}

\author{
Judith F. Baumhauer MD, MPH, Kevin J. Bozic MD, MBA
}

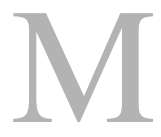

easuring outcomes from the patient's perspective is not a new concept. In 2010, the Harvard Business School economist Michael E. Porter PhD wrote: "Value should always be defined around the customer, and in a well-functioning health care system, the creation of value for patients should determine the rewards for all

A Note from the Editor-in-Chief:

We are pleased to present to readers of Clinical Orthopaedics and Related Research ${ }^{\circledR}$ the latest Value-based Healthcare column (formerly Orthopaedic Healthcare Worldwide). Value-based Healthcare explores strategies to enhance the value of musculoskeletal care by improving health outcomes and reducing the overall cost of care delivery. We welcome reader feedback on all of our columns and articles; please send your comments to eic@clinorthop.org. The authors certify that they, or any members of their immediate families, have no funding or commercial associations (eg, consultancies, stock ownership, equity interest, patent/licensing arrangements, etc.) that might pose a conflict of interest in connection with the submitted article. All ICMJE Conflict of Interest Forms for authors and Clinical Orthopaedics and Related Research ${ }^{\circledR}$ editors and board members are on file with the publication and can be viewed on request.

The opinions expressed are those of the writers, and do not reflect the opinion or policy of $\operatorname{CORR}^{\mathbb{R}}$ or The Association of Bone and Joint Surgeons ${ }^{\circledR}$. other actors in the system. Since value depends on results, not inputs, value in health care is measured by the outcomes achieved, not the volume of services delivered, and shifting focus from volume to value is a central challenge" [4].

Previously, outcome instruments consisted of factors the physician found important and were used primarily for research on a new technique or treatment method. The analysis was completed at the end of the episode-ofcare and the treatment was either deemed successful or not. These physician-derived instruments, however, are being replaced with validated, real-time patient-reported outcomes (PROs). Through the use of PRO measures (PROMs), the patient's

J. F. Baumhauer MD, MPH

Department of Orthopaedics, University of Rochester School of Medicine and Dentistry, Elmwood Avenue, Box 665, Rochester, NY 14642, USA e-mail: Judy_Baumhauer@URMC. Rochester.edu

\section{K. J. Bozic MD, MBA $(\bowtie)$}

Department of Surgery and Perioperative Care, Dell Medical School, University of Texas at Austin, 1400 Barbara Jordan Blvd., Suite 1.114, Austin, TX 78723, USA

e-mail: kevin.bozic@austin.utexas.edu voice is being heard, quantified, and compared to normative data in a large variety of domains such as physical function, pain, depression, anxiety, and fatigue.

The PROMIS (Patient Reported Outcome Measurement Information System) is the product of a USD 100 million NIH initiative aimed at developing valid, precise measurements of a patient's physical, mental, and social health. The various tools in the PROMIS toolkit can help the patient quantify what (s)he is able to do and how (s)he is feeling [5]. The questions can be asked in a paper format or electronically. The real efficiency advantage is in using the computerassisted technology (CAT) and itemresponse theory. This format allows for the followup question to be selected based on the response to the prior question. In this fashion, an available databank of 121 questions can be used selectively, choosing only those questions that best apply, in order to assess a patient's physical function in as few as four to seven questions. This saves time without compromising accuracy or validity. The scores can be imported into an electronic health record for real-time viewing and clinical decision-making (Fig. 1). It is only through the efficient, valid, and instantaneously 


\section{Value-based Healthcare}

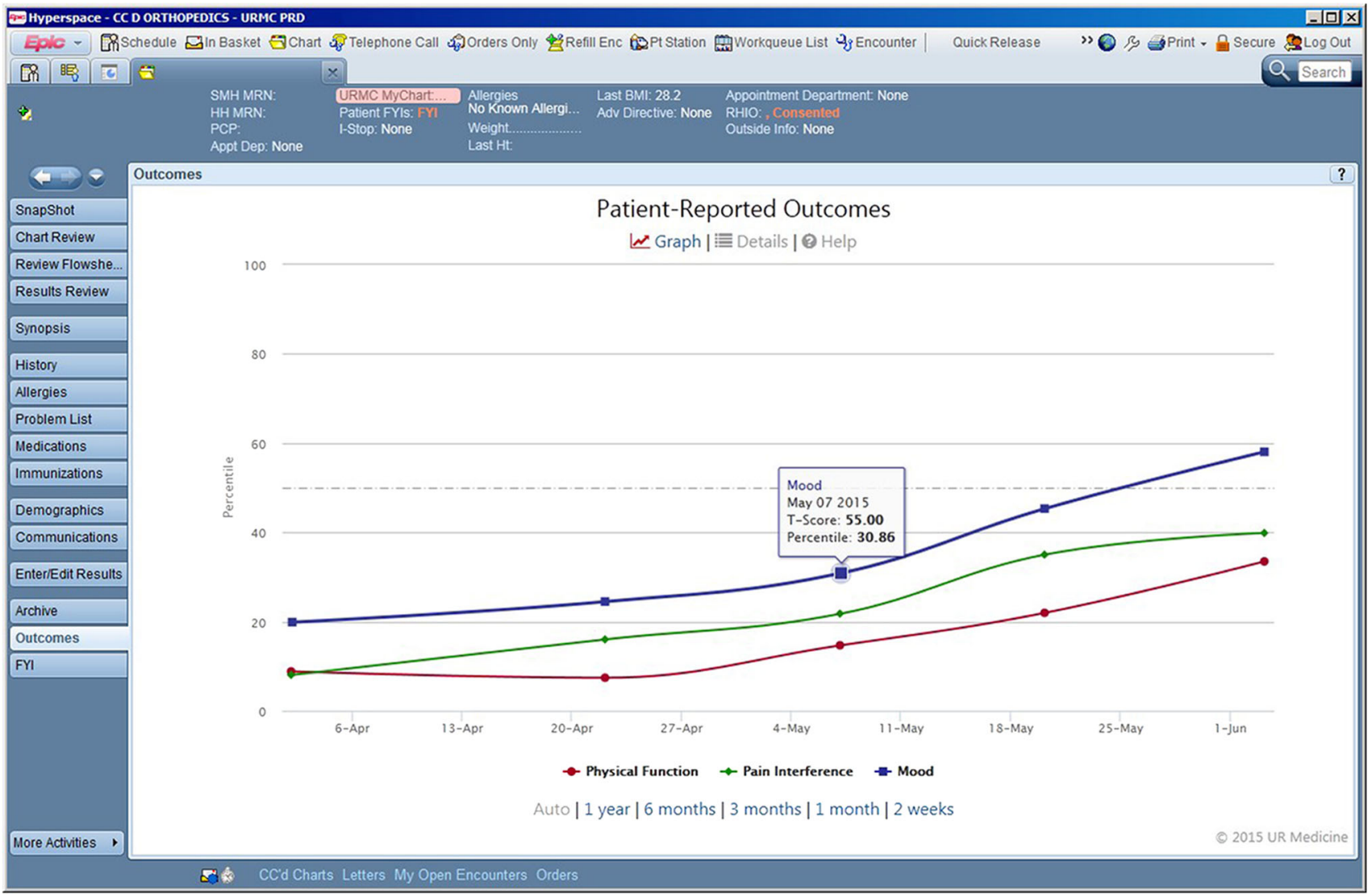

Fig. 1 PROMIS physical function (red), pain interference (green), depression (mood) (blue) t-scores (represented as percentile) for each date of clinic visit viewed in the electronic record. (Published with permission from the University of Rochester Medical Center).

available viewing of outcomes scores compared to a normative population that the paradigm shift in utilizing PRO data for clinical decision-making can occur.

Papuga and colleagues [3] published on more than 100 patients with ACL reconstructions using the PROMIS physical function CAT tool and compared it to the commonly used IKDC Subjective Knee Evaluation Form in a high-volume clinic. Figure 2 shows one of these graphs for the expected physical function after ACL reconstruction surgery. This graph simply displays a curve over time of expected recovery for this knee surgery. This is not hypothetical. This is not ivory-tower stuff. This is realworld useful, and surgeons can-and do-use it right now.

With a value of 50 being normal function, the majority of patients had a value closer to 40 , which is considered impaired. A chart like this may provide comfort to a patient, as he or she will know that they indeed have a problem 


\section{Value-based Healthcare}

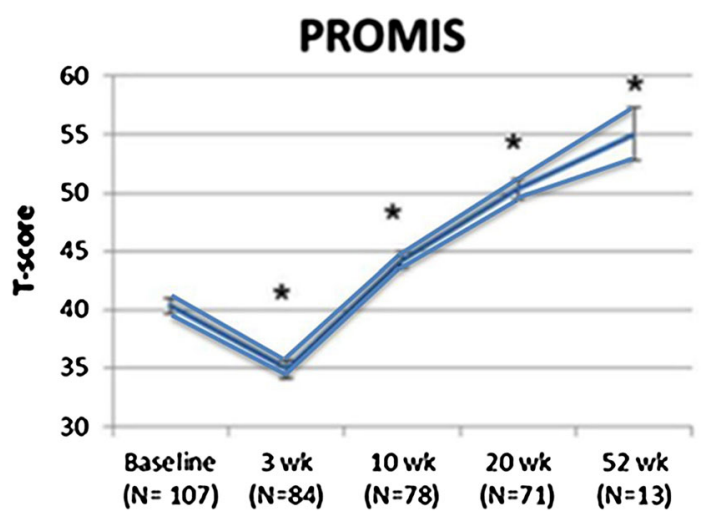

Fig. 2 Baseline and postoperative ACL reconstruction PROMIS physical function t-scores (+/- standard error) over time [3]. (Reproduced with permission from John Wiley \& Sons, Inc).

and the surgery recommendation seems appropriate. This is powerful information. For insurers, it documents that there is a functional deficit and surgical approval appears appropriate.

As the patient recovers from his/her surgery, the PROMIS scores can be compared to an "expected recovery" graph. The patient's own graph can be superimposed on "expected recovery" graph. If the patient does better than expected, he or she could potentially move toward a home exercise program rather than formal physical therapy and save the USD 25 to USD 50 copay. If the patient is doing worse, this might trigger more physical therapy or concerns regarding a complication like an infection, regional pain syndrome, or arthrofibrosis. The data is shared in this graphic display with the patient every visit to help motivate and engage the patient.

As we obtain and analyze PROMs, we have the opportunity to use them in concert with traditional physical exams, patient history, and radiographic imaging to aid in patient selection for surgery. A recent publication [2] utilizing PROMs found higher functioning patients who underwent hip replacement surgery had a lower chance of meaningful clinical improvement. Patients come to surgeons for advice. They want to know, "If I had surgery, would it be worth it?" For higher functioning patients, it might not be. Obtaining PROMs can help us advise our patients when surgery is a good option or not using data and not instinct.

Similarly, mental health issues have been linked to poor patient outcomes.
As orthopaedic surgeons, we have not been in the business of assessing the mental health of our patients, however, if we want to improve patient care, we need to start. PROMs can help us-help our patients.

Sharing the information gleaned from PROMs measured in the office may also improve patient engagement. Patients who read their notes, collect their personal health data, and maintain a record are more engaged, empowered, and activated to participate in their healthcare. One study found that patients with hip and knee arthritis who had higher levels of patient engagement preoperatively were more likely to achieve greater pain relief and satisfaction after total joint arthroplasty [1].

At the University of Rochester Medical Center (URMC) a common core of PROMs (PROMIS physical function, pain interference, and depression CAT assessments) have been collected by orthopaedists for the past year for every patient, every clinic, and every visit [6]. According to the URMC PROMIS database (April 2015February 2016), a total of 58,360 unique orthopaedic patients have been given the PROMIS (94\% administration rate) during 151,532 patient encounters. A total of 366,280 PROMIS scores have been collected with 1.9 million questions asked and answered [6]. These data are provided to patients to facilitate 


\section{Value-based Healthcare}

shared clinical decision making regarding treatment options.

Once the process of data collection and efficiency was maximized by URMC Orthopaedics, the rest of the ambulatory clinical services at URMC were offered the common core, in addition to other PROMIS CAT tools. To date, more than 20 departments and divisions have begun the process of PROMIS data collection. With the tremendous interest in the PROMIS program, the plan is to have all ambulatory clinical services at URMC participating by the end of 2017. The shared PROMIS scores will be visible on a commonly accessible graph in the electronic record, and our patients' physical function, pain, and emotional health can be followed jointly over time by members of the healthcare team. We expect this approach will provide treatment curves to predict patient outcomes.

Thinking broadly, this approach reaches far beyond shared clinical decision making with individual patients. A unified healthcare PROMs program can be linked with other health data and aid in population health management, provider performance assessment, comparative effectiveness evaluations, and value-based community initiatives.

PROs are not a new concept; however, the ability to collect PRO data in real time, and the focus on sharing this information with the patient and using it in clinical decision making is the future. Rather than assessing outcomes at the conclusion of treatment, and using it primarily for research purposes, the goal is to have PROMs discussed with the patient during their office visit, compared to normative values for similar conditions, and for the patient and his or her healthcare team to use this data to inform clinical decision making consistent with the patient's preferences, values, and severity of disease.

\section{References}

1. Andrawis J, Akhavan S, Chan V, Lehil M, Pong D, Bozic KJ. Higher preoperative patient activation associated with better patient-reported outcomes after total joint arthroplasty. Clin Orthop Relat Res. 2015;473: 2688-2697.

2. Berliner JL, Brodke DJ, Chan V, SooHoo NF, Dozic KJ. John Charnley award: Preoperative Patient-reported outcome measures predict clinically meaningful improvement in function after THA. Clin Orthop Relat Res. 2016;474:321-329.

3. Papuga MO, Beck CA, Kates SL, Schwarz EM, Maloney MD. Validation of GAITRite and PROMIS as high-throughput physical function outcome measures following ACL reconstruction. $J$ Orthop Res. 2014;32:793-801.

4. Porter ME. What is value in health care? N Engl J Med. 2010;363:24772481.

5. Pupil Records Online Management Information System. Welcome to PROMIS. Available at: https://www.mypro mis.org/. Accessed March 4, 2016.

6. University of Rochester Medical Center. Orthopaedics and rehabilitation. Available at: https://www.urmc.roch ester.edu/orthopaedics.aspx. Accessed March 22, 2016. 